\title{
Electric field distributions in CdZnTe due to reduced temperature and $x$-ray irradiation
}

\author{
P. J. Sellin, ${ }^{1, \text { a) }}$ G. Prekas, ${ }^{1}$ J. Franc, ${ }^{2}$ and R. Grill ${ }^{2}$ \\ ${ }^{1}$ Department of Physics, University of Surrey, Guildford GU2 7XH, United Kingdom \\ ${ }^{2}$ Institute of Physics, Charles University Prague, Prague CZ-121 16, Czech Republic
}

(Received 12 February 2010; accepted 1 March 2010; published online 1 April 2010)

\begin{abstract}
Real-time Pockels imaging is performed on semi-insulating CdZnTe to measure the electric field profile in the material bulk. In steady-state room temperature conditions the measured electric field profile is uniform, consistent with a low space charge concentration. At temperatures $<270 \mathrm{~K}$ a significant nonuniform electric field profile is observed, which we explain in terms of temperature-induced band bending at the metal-semiconductor interface, causing the formation of positive space charge in the bulk. Similar electric field distortion effects are observed when room temperature CdZnTe is irradiated by x-rays, causing a high rate of photoinduced charge injection.

(C) 2010 American Institute of Physics. [doi:10.1063/1.3373526]
\end{abstract}

Cadmium zinc telluride (CdZnTe) is a wide bandgap II-VI semiconductor material that has been studied extensively for use as an $\mathrm{x}$-ray and gamma ray detector. The attraction of CdZnTe for this application is due to the high $\mathrm{x}$-ray and gamma photon attenuation coefficient, caused by the large effective atomic number, combined with good charge transport properties. In addition, the bandgap of $\mathrm{CdZnTe}$ is approximately $1.6 \mathrm{eV}$ (for $10 \% \mathrm{Zn}$ concentration) which prevents excessive thermal generation of carriers at room temperature and so allows room temperature detector operation without excessive dark current. The availability of high purity semi-insulating CdZnTe has improved in recent years, with material grown by a range of methods including high pressure Bridgman, traveling heater method, and vapor phase transport. In the highest quality material, the bulk resistivity is in excess of $10^{10} \Omega \mathrm{cm}$, with a correspondingly low free carrier concentration. The material is semiinsulating due to self-compensation due to one or more deep mid-bandgap states in equilibrium with shallow impurity levels. At room temperature the electron transport in $\mathrm{CdZnTe}$ is predominantly unaffected by the presence of deep levels, with a room temperature electron drift mobility of $1000 \mathrm{~cm}^{2} / \mathrm{V} \mathrm{s}$. The dominant mobility mechanisms are a combination of phonon scattering and impurity scattering, and the electron mobility increases with decreasing temperature, e.g., reaching $1500 \mathrm{~cm}^{2} / \mathrm{V} \mathrm{s}$ at $250 \mathrm{~K} .{ }^{1}$ In contrast hole transport is relatively poor, with hole drift mobility values of $80-120 \mathrm{~cm}^{2} / \mathrm{V} \mathrm{s}$ reported at room temperature. In practice, the mean hole drift length is lifetime limited, and is significantly reduced in comparison to the mean electron drift length.

In this work, we have studied the distribution of the electric field profile through the bulk of $\mathrm{CdZnTe}$ radiation sensors using the Pockels electro-optical effect. The aim of this study is to investigate the development of nonuniform electric field distributions which occur when the device is operated at low temperature or under conditions of heavy $\mathrm{x}$-ray irradiation. Under normal operation the bulk electric field is constant as a function of depth, consistent with a low concentration of ionized space charge in the material. When

${ }^{a)}$ Electronic mail: p.sellin@surrey.ac.uk. operated at low temperature, various authors have reported a degradation in the measured gamma energy resolution, due to a deterioration in the charge carrier drift properties in the device. This is contrary to the expected improvement in drift mobility that exists under moderate cooling (e.g., temperatures down to $200 \mathrm{~K}$ ). Pockels measurements have been used to directly demonstrate the presence of a nonuniform electric field profile in devices at temperatures below $250 \mathrm{~K}$ that is the cause of the poor detector response.

The Pockels electro-optical effect has been used extensively to measure internal field distributions in various material systems which exhibit voltage-dependent birefringence ${ }^{2,3}$ In our work, samples of CdZnTe were supplied by Yinnel Tech Inc., grown by a modified vertical Bridgman method. The resistivity of the sample was $>10^{10} \Omega \mathrm{cm}$, and the electron mobility-lifetime product was $>5 \times 10^{-3} \mathrm{~cm}^{2} / \mathrm{V}$. The device was fabricated with planar evaporated gold contacts on the top and bottom surfaces, with a high quality optical polish applied to the side surfaces of the device. The CdZnTe sample was mounted on a temperature-controlled cold finger inside a custom optical cryostat. The cryostat is fitted with IR optical windows, and the sample is illuminated with low intensity IR light which has passed through a narrow band-pass $980 \mathrm{~nm}$ filter. A pair of $90^{\circ}$ orthogonally orientated polarizing filters are arranged either side of the cryostat, with the transmitted light imaged by an IR-enhanced digital charge coupled device camera. Under these conditions the intensity of the transmitted light $I(x, y)$ has the following form:

$$
I(x, y)=I_{0}(x, y) \sin ^{2}\left[\frac{\sqrt{3} \pi n_{0}^{3} r_{41} d}{2 \lambda_{0}} E(x, y)\right],
$$

where $I_{0}(x, y)$ is the maximum intensity with the polarizers parallel, $n_{0}$ is the zero-field refractive index in CdZnTe, $r_{41}$ is the linear electro-optic coefficient, and $d$ is the optical path length through the crystal $(6 \mathrm{~mm})$. Very long acquisition times were used to acquire optical transmission images through the CdZnTe as a function of bias voltage, using a very low optical power of $900 \mu \mathrm{W} / \mathrm{cm}^{2}$ incident on the sample. 


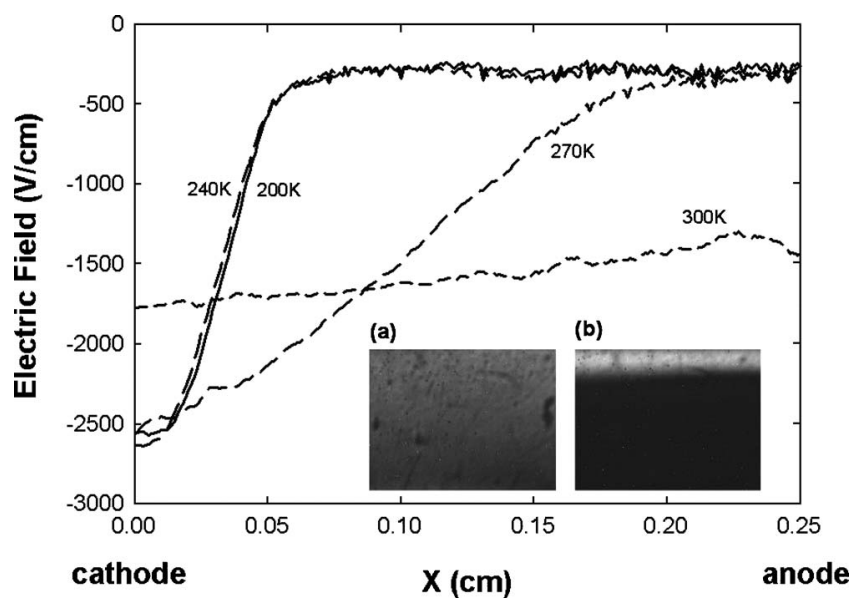

FIG. 1. Electric field profiles measured from the CdZnTe device at low temperature, with a voltage of $500 \mathrm{~V}$. The inset figures show the Pockels image acquired at (a) $300 \mathrm{~K}$ and (b) $240 \mathrm{~K}$.

Figure 1 shows the experimental electric field profiles measured in $\mathrm{CdZnTe}$ as a function of temperature. The inset images (a) and (b) show the optical image obtained at $300 \mathrm{~K}$ and $240 \mathrm{~K}$, respectively. At $300 \mathrm{~K}$ the optical image shows a uniform illumination from the top of the image (cathode electrode) to the bottom of the image (anode electrode), equal to a uniform electric field profile. At $240 \mathrm{~K}$ the optical image shows a band of high transmission intensity close to the cathode, signifying a region of high electric field. The main figure shows the resulting electric field profiles, with a strong transition between 270 and $240 \mathrm{~K}$ to a nonuniform distribution. At low temperatures the device shows a high field region which extends $0.5 \mathrm{~mm}$ from the cathode, followed by a low field region in the remainder of the device which is the cause of the poor charge drift at low temperature.

The observed temperature dependences of electric field profiles can be explained by the accumulation of space charge at deep levels. In this case, the most simple model consists of one near-midgap state, which is pinning the Fermi level. The experimentally observed low temperature evolution of the electric field (Fig. 1) can be explained by an increase in the difference between work functions of $\mathrm{Au}$ and CdTe with decreasing temperature. Gold represents one of the most optimal materials for formation of noninjecting and nonblocking contacts on semi-insulating CdTe and CZT. The work function of $\mathrm{Au} \Phi_{\mathrm{Au}}$ is $\sim 5.3 \mathrm{eV}$ (Ref. 4) while the work function in semi-insulating $\mathrm{CdTe}$ is in the range of 5.2-5.4 eV (Ref. 5) depending on the position of the Fermi level in the near midgap region. We assume that the work function difference is nearly zero at room temperature (the profile of the field is almost flat, see Fig. 1). With decreasing temperature the CZT bandgap increases, according to Refs. 6 and 7.

Consequently the near midgap level shifts as the bandgap increases. We assume in the model, that the midgap level which is pinning the Fermi level moves up in the absolute energy scale to the conduction band, so increasing the difference between the work function of metal and semiconductor $\left(\Phi_{\mathrm{M}}>\Phi_{\mathrm{S}}\right)$. This results in the formation of positive space charge near the electrodes (bands are bent upwards). The contact, which is nearly ohmic at $300 \mathrm{~K}$ becomes slightly Schottky, when the temperature decreases. In our model, we

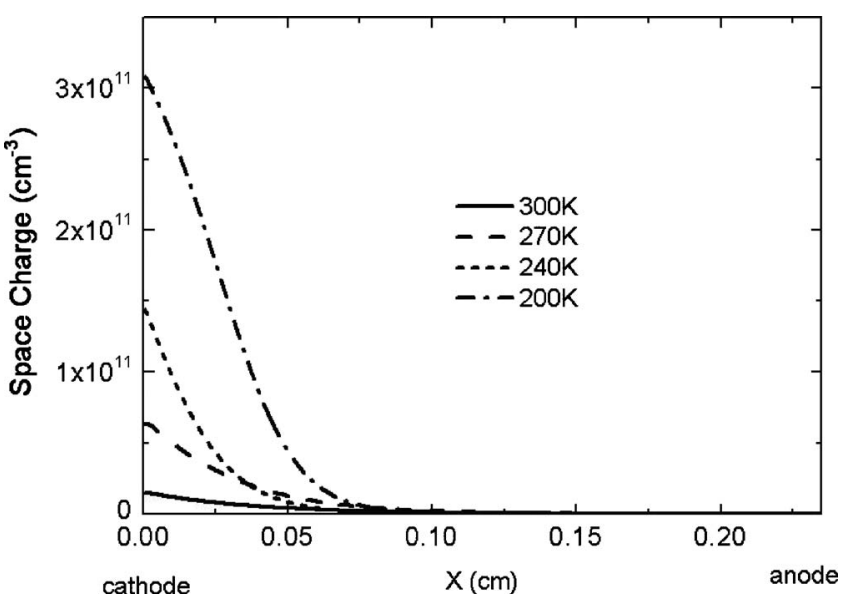

FIG. 2. Profile of the calculated space charge as a function of temperature.

assume that the shift is $20 \mathrm{meV}$-part of the total bandgap change which is approximately $30 \mathrm{meV}$ in the range 200 $300 \mathrm{~K}$. The parameters of the deep level used in the simulations are as follows: $\mathrm{N}_{\mathrm{t}}=1 \times 10^{12} \mathrm{~cm}^{-3}, \mathrm{E}_{\mathrm{t}}=\mathrm{E}_{\mathrm{c}}-0.7 \mathrm{eV}, \sigma_{\mathrm{e}}$ $=5 \times 10^{-13} \mathrm{~cm}^{2}$, and $\sigma_{\mathrm{h}}=3 \times 10^{-14} \mathrm{~cm}^{2}$, where $\mathrm{N}_{\mathrm{t}}$ is the concentration of the deep level, $\mathrm{E}_{\mathrm{t}}$ is the energy calculated from the conduction band, and $\sigma_{\mathrm{e}}$ and $\sigma_{\mathrm{h}}$ are the electron and hole capture cross-sections. Simultaneous solution of the drift-diffusion and Poisson equations was used to calculate the resulting profiles of the space charge and of the electric field at temperatures between 200 and $300 \mathrm{~K}$.

The profile of the space charge in dependence of the temperature is shown in Fig. 2. The positive space charge increases with increasing band bending, extending to approximately $20 \%$ depth of the sample.

The corresponding profiles of electric field are shown in Fig. 3. These profiles and their temperature development agree very well with the experimental data (Fig. 1) demonstrating dramatic changes of the electric field due to space charge present in the detector as a result of small band bending at the metal/semiconductor interface. Deformation of electric field can result in a decrease in charge collection efficiency. The weakening of electric field below the anode increases the collection time of electrons $\tau_{\text {col }}$. The probability of electron trapping and recombination increases causing a deterioration in the detector performance due to incomplete charge collection.

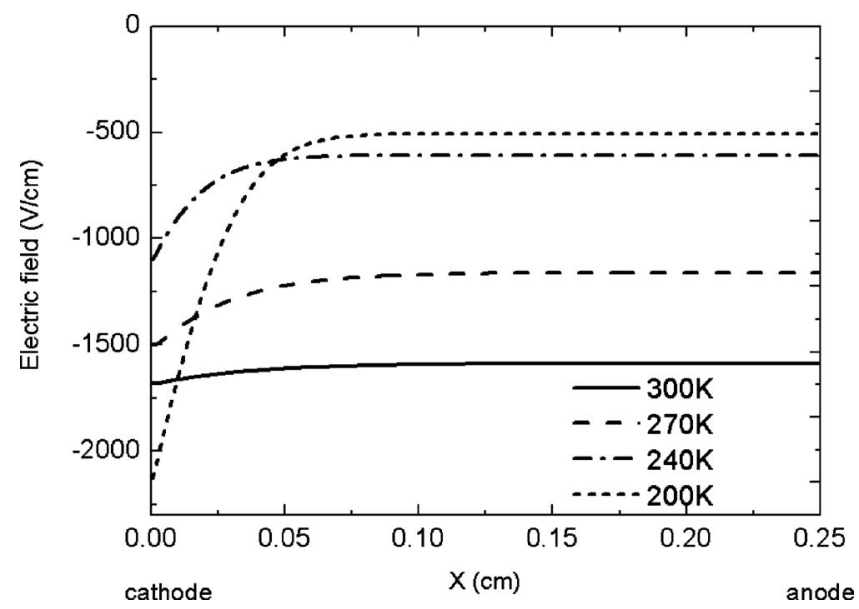

FIG. 3. Profile of the calculated electric field as a function of temperature 


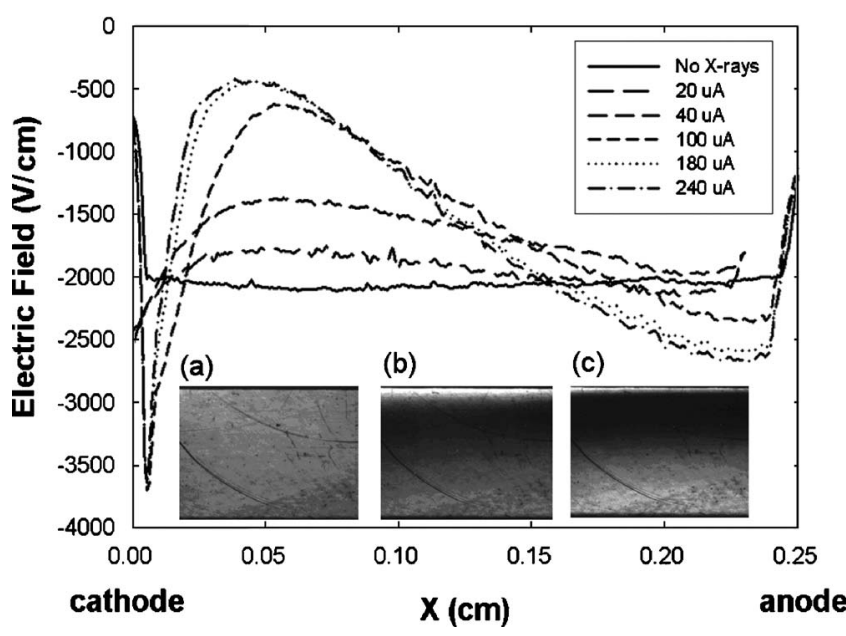

FIG. 4. X-ray induced electric field profiles, at room temperature. The data were acquired at dose rates of 0 to $5 \mathrm{mGy} / \mathrm{min}$. The inset Pockels images were acquired at (a) $0 \mu \mathrm{A}$, (b) $80 \mu \mathrm{A}$, and (c) $480 \mu \mathrm{A}$ while the bias on the crystal was constant at $-450 \mathrm{~V}$.

A similar modification of the internal field profile is seen in CdZnTe under conditions of high charge injection, even at room temperature. ${ }^{8}$ The spectroscopic performance of $\mathrm{CdZnTe}$ detectors is often observed to deteriorate when, for example, irradiated with a high intensity x-ray flux. This behavior can substantially affect the performance of $\mathrm{CdZnTe}$ detectors when used in X-ray imaging equipment. In situ Pockels imaging of a CdZnTe detector has been carried out while simultaneously irradiating the device with $\mathrm{X}$-rays. Figure 4 shows the resulting electric field profiles, acquired at a fixed bias of $-450 \mathrm{~V}$ and room temperature, during $\mathrm{x}$-ray irradiation with a $50 \mathrm{kV}$ x-ray tube. The dose rate on the sample was adjusted as a function of the x-ray tube current from 0 to $5 \mathrm{~Gy} / \mathrm{min}$. At increased dose rate a significant alteration to the electric field profile is observed, which produces a local region of high field close to the irradiated cathode, and a field minimum approximately $20 \%$ into the device depth. This behavior is consistent with the build up of positive space charge caused by photogenerated holes which are strongly trapped in deep levels in the material. ${ }^{9}$ At high fluence irradiation, the rate of charge injection into the $\mathrm{CdZnTe}$ is sufficiently high that charge trapping dominates over thermal emission and recombination processes, and a significant net space charge accumulates in the bulk.

This work is part funded by the UK Basic Technology "HEXITEC" program, and the Ministry of Education of the Czech Republic (research plan MSM 0021620834).

${ }^{1}$ K. Suzuki, S. Seto, T. Sawada, and K. Imai, IEEE Trans. Nucl. Sci. 49, 1287 (2002).

${ }^{2}$ A. Cola, I. Farella, A. Mancini, W. Dusi, and E. Perillo, Nucl. Instrum. Methods Phys. Res. A 568, 406 (2006).

${ }^{3}$ A. Burger, M. Groza, Y. Cui, D. Hillman, E. Brewer, A. Bilikiss, G. W. Wright, L. Li, F. Fu, and R. B. James, J. Electron. Mater. 32, 756 (2003). ${ }^{4}$ CRC Handbook on Chemistry and Physics, edited by D. R. Lide (CRC, New York, 1999), pp. 12-124.

${ }^{5}$ A. W. Brinkman, Properties of Narrow Gap Cadmium based Compounds, EMIS Datareviews Series No. 10 (Inspec, London, 1994), p. 575.

${ }^{6}$ J. Franc, P. Hlídek, P. Moravec, E. Belas, P. Höschl, L. Turjanska, and R. Varghová, Semicond. Sci. Technol. 15, 561 (2000).

${ }^{7}$ J. P. Laurenti, J. Camassel, A. Bouhemadou, B. Toulouse, R. Legros, and A. Lusson, J. Appl. Phys. 67, 6454 (1990).

${ }^{8}$ D. S. Bale, S. A. Soldner, and C. Szeles, Appl. Phys. Lett. 92, 082101 (2008).

${ }^{9}$ D. S. Bale and C. Szeles, Phys. Rev. B 77, 035205 (2008). 